\title{
artículos
}

\section{Breve reseña histórica de la CDU en España}

\section{Rosa San Segundo Manuel}

ProfesoraTitulardela Universidad de la AsignaturaSistemas deClasificación,en la Diplomatura de Biblioteconomía y Documentación de la Universidad Carlos III de Ma drid, Es pa ña

\section{RESUMEN}

$\boldsymbol{L}$ a CDU ha sido el sis te ma im plan ta do en Es pa ña de for ma to tal en to das las bi blio te cas de titularidad es ta tal. A partir del 1895, año en que se ce le bra la Pri me ra Con fe ren cia In ter na cio nal de Bi bliografía y que el Sistema Decimal empieza a ser implantado, mayormente, en el ámbito europeo, co mien za dar se a co no cer en Es pa ña. Así, la in tro duc ción de la CDU en Es pa ña va a es tar su je ta a nume ro sas di fi cul ta des, y se va a pro du cir, en un pri mer mo men to, por he chos ais la dos de bi blio te carios, y en un mo men to pos te rior va a con tar con el apo yo de la ad mi nis tra ción es pa ño la. Será en 1939 cuan do se im plan te de for ma ofi cialla CDU en to das las biblio te cas es pa ñolas. Final men te a par tir de 1989 se de ro ga la obli ga to rie dad por par te de los bi blio te ca rios es pa ño les de em plear la CDU para cla si fi car los fon dos y los ca tá lo gos.

\section{ABSTRACT}

$\boldsymbol{T}$ he Uni ver sal De ci mal Clas si fica tion has been the sys tem im po sed upon all sta te-run libraries in Spain. Sin ce 1895, the year in which the First In ter na tio nal Bi blio graphy Con fe ren ce washeld, and when the De ci mal Sys tem be gan to be in tro du ced main ly in Eu ro pean cir cles; it also started to be divul ged in Spain. The in tro duc tion of the UDC in Spain was sub ject to nu me rous diffi cul ties and at first was the work of in di vi dual li bra rians, though la ter on it would have the support of the spa nish admi nis tra tion. It was in 1939 when the UDC was offi cia lly im po sed on all spa nish li bra ries. Finally, in 1989, it was de cla red non- o bli ga tory for spa nish li bra rians to uti li ze the UDC for the clas sifiction of ar chi ves and ca ta logues.

\section{PRIMEROSINTENTOS DE DIFUSION}

\section{DE LA CDU EN ESPAÑ}

1 primer intento de difusión en España de Cla Clasificación Decimal, tras la celebración en 1895 de la PrimeraConferencia Internacio nal de Biblio gra fía, or ga ni za da por elentonces denominado y recién creado Instituto Internacional de bibliografía, ${ }^{1}$ fue abordado por el bibliotecario de la Uni ver si dad de Sa lamanca ManuelCastillo, quien por ello sufrió grandes derrotas y fue rápidamente apartado de su car go de bi blio te ca rio, pa san do de nue vo a ejercer susfun cio nes de ca te drático.

Manuel Castillo fue el primer difusor y traductor de la Clasificación Decimal en España. El tuvo co no ci mien to de la ce le bra ción de 1a. Conferencia Bibliográfica Internacional en 1896, a través de una reseña referente al
Ins ti tu to apa re ci da en una re vis ta fran ce sa de jurisprudencia, ${ }^{2}$ al mismo tiempo que tuvo conocimiento de la Clasificación Decimal. No dudó en di fun dir las ven ta jas del sis te may en hacerse su transmisor. Castillo fue el primer miem broes pa ñol y co la bo ra dor del Ins titu to, ade más de ha cer una pri me ra tra duc ción de las tablas.

Los ale ga tos pos te rio res a fa vor de la Cla si fica ción De ci mal fue ron te nues ante la gran derro ta que ha bía su fri do di cho sis te ma, ya que se ha bía de se cha do, y en las bi blio te cas no se implantaba. Esta situación estuvo motivada, en tre otrascau sas, por que Es pa ña atra ve saba momentos difíciles. Y la situación política, eco nó mi ca y so cial pre sen ta ba se rias dificul tades como para adoptar o aceptar cualquier injerencia extranjera en los asuntos nacionales. Así, el Go bier no de la Res tau ra ción, bajo la presidenciaalternativadel con servadorCanoras y del liberal Sagasta (1876-1898), no mantuvo ni practicó política exterior, lo que revirtió en un aislamiento de España como respuesta alusiva a las dificultades internacionales. Ello también repercutió en la organi za ción téc ni ca de las bi blio gra fías y bi bliotecas; éstas atravesaron un período de gran ais la mien to y no in cor po ra ron los nue vos métodosbibliográficos.

Sí es cier to, sin em bar go, que a par tir de la di ficultad de la Clasificación Decimal abordada por Castillo se inicia una mayor dedicación y preocu pación por la me to do logía a em plearen

1 Hoy bajo la denominación de FID, Federación Internacional de Documentación 
la ela bo ra ción de los ca tá lo gos de las bi blio tecas. Aunque la Clasificación Decimal no se adoptó rápidamente, sí marcó una impronta en las tareasbibliográfico-bibliotecarias.

El se gun do gran in ten to por im plan tar la CDU en las bibliotecas españolas fue llevado a efecto por la Biblioteca de Ingenieros militares, que igualmente se tropezó con serias di ficul ta des. Aún al ini cio del si glo XX el Instituto Internacional de Bibliografía no era muy conocido entre los bibliotecarios españo les. Di cha Bi blio te ca co la bo ró con el Ins titu to y se hizo miem bro en 1908 ; creó un ser vicio de información bibliográficamediante el cual trató de proporcionar la información exis ten te en li bros, pe rió di cos y re vis tas re lati va a te mas afi nes a la in ge nie ría. Asi mis mo, este nuevo servicio ofrecía información de todo lo ex pues to en la Revuedel'Inge nieuret index technique, publicación que formaba parte de la Bibliografía Uni versaldel Ins ti tur to Internacional de Bibliografía. Leopoldo Giménez, Capitán de Ingenieros, estudió la ClasificaciónDecimal y tra du jo las ta blas de la CDU re fe ren tes a la In ge nie ría, o sea, el núme ro 62, e in cluía una de ta lla da ex plici ta ción de las sub di vi sio nes de este nú me ro y, asi mismo, in for mó so bre el nue vo ser vi cio de in formación de la biblioteca en su obra. ${ }^{3}$ Debemos, pues, con cluir que la im por tan cia de esta biblio te ca, in ser ta en una his to ria de la cla si fi cación biblioteco-bibliográfica en España, ra di ca en ser la pri me ra que im plan ta el Sis tema Decimal y en ser miembro del Instituto, aun que no son sus colabora do res los prime ros difusores de la existencia y la actividad del Ins ti tu to en Es paña. ${ }^{4}$

El siguiente gran propulsor de la acción del Ins ti tu to fue el In ge nie ro de Mon tes Ricardo Codorniú, que se preo cu pó por la pro ble mática de la Clasificaciónbibliográfica y publicó en 1911 una mo no grafía so bre la Cla si ficación Decimal. ${ }^{5}$ En ella tradujo los números de las tablas relativos al área temática de
Montes. Codorniú fue miembro del Instituto des de $1908^{6} \mathrm{y}$, por tan to, des de esta fe cha reci bió el Bo le tín del Ins ti tu to. Ello le per mi tió ser buen conoce dor de la Cla si fi ca ción De cimal y de la ac ti vi dad de aquél. Por con se cuencia, Co dor niú des ta ca como pio ne ro al tra dur cir y difundir las tablas del SistemaDecimal en Es pa ña, aun que su la bor como miem bro y propa gan dis ta del Ins ti tu to hay a es ta do to tal mente olvidada.

Hubo, en un mo men to pos te rior, otros bi bliotecarios que de forma aislada propagaron la CDU, como el Bibliotecario Román Gómez Villafranca, que tra ba jó en la Bi blio te ca Provin cial del Ins ti tu to Ge ne ral y Téc ni co de Badajoz y fue, también, uno de los primeros miembros españoles del Instituto. Su labor mas des ta ca da, en la di fu sión de la Cla si fi ca ción De ci mal, fue la rea li za ción de un ín di ce bibliográfico de la Revista de Archivos, Bibliotecas y Museos, en 1911, haciendo uso del Sistema Decimal. ${ }^{7}$ Ello le convirtió en uno de los prime ros in tro duc to res del Sis te ma De ci mal des de una pra xis bi blio gráfica, y fue uno de los primeros bibliotecariosque se enco men da ron en la ta rea de di fun direl Sis te ma Decimal.

La implantación de la CDU en España fue muy len ta, ade más las bi blio te cas eran es casas y carecían de directrices para realizar los trabajos téc ni cos. En Ca ta lu ña, fren te al res to de Es paña, las bi blio te cas han te ni do una evolución his tóricadiferente, Portanto, podemos re se ñar que en Ca ta lu ña la si tua ción de la clasificación ha te ni do, tam bién, una tra yec to ria distinta. Aquí destacaelbibliotecario Sebastián Far nés, que rea li zó en 1914 una ex pli citación del siste maclasifica torioem pleadoen el catálogo metódico de esta biblioteca. Por vez pri me ra se em plea la Cla si fi ca ción De cimal en el ám bi to ca ta lán. Este ca tá lo go me tódi co lo hizo me dian te la apli ca ción de la Clasificación Decimal. Postuló la adopción de este Sistema no sólo desde apoyaturas teóri- cas, sino también desde la práctica, ya que, ade más de apli car el sis te ma al ca tá lo go de la bi blio te ca, tra du jo las ta blas al cas te lla no y se consi de ró erró nea men te el pri mer tra duc tor. ${ }^{8}$ Sin em bar go, sí que fue el pri me ro en mo di ficar el número de las CDU correspondiente a la len gua ca ta la na. La no ta ción re la ti va a este ámbito en la CDU era la siguiente:

\begin{tabular}{||l|l||}
\hline Francia & 44 \\
\hline España & 46 \\
\hline Cataluña & 449.9 \\
\hline
\end{tabular}

La mo di fi ca ción de Far nés fue de bi da a que si el 4 representa a Europa, el 6 a la Península Ibé ri ca y el 7 a la par te orien tal de la mis ma, y si la sub di vi sión geo gráfica coin ci de con la fi lo ló gi ca, porcon secuen cia, la len gua ca ta la na no podrá ser representada por el número 449.9 como aparece en la CDU, ya que esto supone considerar a la lengua catalana como una va rian te de la len gua fran cesa, portan to, según Farnés, la lengua catalana deberá ser representada por el 467. Este será el motivo que ori gi na rá que en Ca ta lu ña no se im plan te más adelante la CDU, sino la Clasificación De ci mal en su ver sión eu ro pea de 1905. Farnés toma las tablas del Sistema Decimal del Manuel del Repertoire Bibliografique Universel con sus au xi lia res de for ma, lu gar, lengua y tiempo, pero modifica el número asignado a la lengua catalana sin dudar de su corrección, ${ }^{9}$ as pec to que re co ge rá, en un momento posterior, el destacado bibliotecario

\section{Jor di Ru bió i Ba la guer.}

La de fen sa del ca ta lán den tro de las ta blas de Clasificación Decimal la inicia Sebastián Farnés con la apoyatura de la reciente creación de un gobierno con cierta autonomía en Cataluña, denominado Mancomunidad de Ca ta lu ña (el 6 de abril de 1914). El sen ti miento na cionalis ta ca ta lán se mani fes tó, de for ma notable, en la organización de la Biblioteca

2 CASTILLO QUIJADA, Manuel. "Una gran adquisición para la bibliografía moderna. La Clasificación Decimal Dewey". En: BOLETIN de Archivos, Bibliotecas y Museos. 1986, año I, n. 56 ; p. 68-72.

3 GIMENEZ, Leopoldo. Noticia sobre el servicio de Información Bibliográfica establecido en la Biblioteca de Ingenieros del Ejército .- Madrid : [s.n.], 1906. Las tablas de la Clasificación base para este servicio sufrieron numerosas modificaciones respecto a las del Sistema Decimal, ya que incluían nuevos términos útiles para este servicio. Con ello se trataba de expresar conceptos y términos nuevos.

4 Así lo consideraba erróneamente Luis de Urzais en el prólogo de la obra de Leopoldo Jiménez, quien no dudó en difundir que el General Marva fue quien por vez primera propagó noticias del Instituto en España.

5 CODORNIU, Ricardo. Clasificación bibliográfica decimal y extracto de las tablas empleadas en el Repertorio Bibliográfico Universal para el uso del personal Facultativo de Montes. Madrid: Imprenta alemana, 1911 ; p.5. Reseñada en: BULLETIN de I'Institut International de Bibliographie, 1911 ; p. $110-198$.

6 BULLETIN de I'Institute International de Bibliographie. 1901, VI ; p. 243-249.

7 GOMEZ VILlafrende, Román. Catálogo de la Revista de Archivos, Bibliotecas y Museos en sus tres épocas (enero de 1871, diciembre de 1910) formado, aplicando la Clasificación Decimal. Madrid, 1911. Este índice abarcaba desde el inicio de la Revista, en 1871, hasta la fecha de publicación del mismo en 1911. A través de la organización sistemática del vaciado de los artículos de la Revista, Gómez Villafranca dio a conocer, de forma muy práctica, la utilidad y practicidad del nuevo sistema clasificatorio. También empleó el Sistema Decimal para la realización del índice de la revista La España moderna.

8 FARNES, Sebastián. Clasificación de la Biblioteca del Fomento del Trabajo Nacional ; p. VI. "No ofrecemos al Fomento una obra original sino una adaptación. No es de despreciar, sin embargo, nuestra diligencia al traducir del francés al castellano lo que no hemos visto hasta ahora traducido".

9 FARNES, Sebastián. Clasificación de la biblioteca del Fomento del Trabajo Nacional. Op. cit.; p. IX-X. 
del Fo men to. El proble ma plan tea do por Farnés desde esta Biblioteca del Fomento conservará su latencia durante el siglo XX y la CDU no será im plan ta da en las bi blio te cas catalanas, sino la Clasificación Decimal desarrollada en el Repertoire y con las modificaciones relativas a la lengua catalana.

Con la implantación del Sistema Decimal, como ya vi mos, en el Servi cio de In for ma ción de la Bi blio te ca de In ge nie ros del Ejér ci to, la Clasificación Decimal va a iniciar un incipiente desarrollo en España. El bibliotecario An to nio Paz y Meliá, ads cri to a la Biblio te ca $\mathrm{Na}$ cio nal de Ma drid en el car go de Jefe de segunda, estudió la incidencia de la aplicación de laCla si ficación De ci mal en bi blio te cas de carácter nacional. ${ }^{10} \mathrm{Se}$ mostró abierto partida rio de laim plan ta ción del Sis te ma De ci mal en las bi blio te cas na cio na les, ya que con sideró que éstas son las principales partícipes en la rea li za ción de las bi blio gra fías na cio na les. Paz y Meliá instó al Gobierno español para que se ad hi rie ra a los pro pó si tos del men cionado Instituto. $Y$ en repetidas ocasioneshizo in fruc tuo sas pe ti cio nes para lo grar la co la boración es pa ñola en ma te riabiblio gráfica; $\mathrm{Paz}$ y Me liá te nía y a 68 años cuan do pu bli có su artículo y todavía estaba en activo. Muere en 1927 , mo men to en que la CDU se en con tra ba lejos de ser implantada.

Es tas pe ti ciones habían sido de ne ga das, pues además, durante la monarquía de Alfonso XIII, Es pa ña se vio so me ti da a un ais la mien to ya practicado en el último tercio del siglo XIX, y que ya ha bía sido pues to de ma ni fies to por la Generación del 98, lo que también se hubo de manifestar en la reiterada negativa del Gobierno español para cooperar con el Instituto. Paz y Meliá consideraba que debía ser el Go bier no quien adop ta ra la de ci sión de implantar el Sistema Decimal, pero no sólo de bía de adop tar esta de ci sión, sino que también debiera colaborar con material y dinero para apoyar el desarrollo de la Clasificación Decimal. Paz y Meliá descalificó el sistema de Bru net, ${ }^{11}$ que fue du ran te mu chos años el modelo clasificatorio para gran parte de los bibliotecarios, españoles, europeos e incluso la tinoamericanos. Abogó por la adop ción del

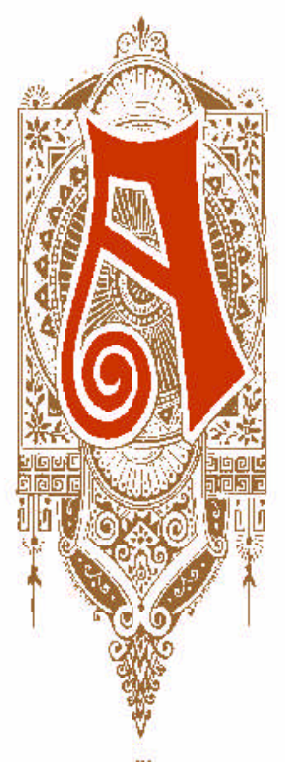

\section{“En las postrimerías del siglo XIX y al inicio del siglo XX los apoyos para la implantación de la CDU en España fueron hechos aislados"}

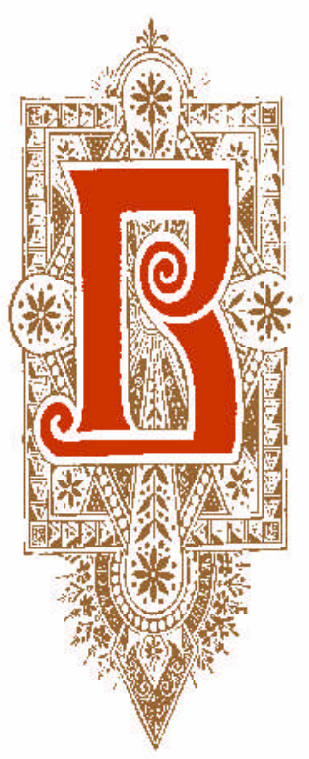

Sis te ma De ci mal, ${ }^{12}$ ya que re por ta ría un gran desarrollo del ámbito bibliográfico español.

Otro difusor en Es pa ña de la CDU fue el padre je sui ta,Ju lián de Eguía, quien pu bli có en 1920 su obra Mibiblioteca, cuyo pri mer tomo titulado Clasificación era una exposición de la Clasificación Decimal. Eguía fue partidario de la im plan ta ción del Sis te ma De ci malen las biblio te cas delEsta does pañol.Las clasificaciones ideadas con anterioridad las consideró inservibles para la organización de los fondos y catálogos de las biblio te cas. ${ }^{13}$

\section{LA ASAM BLEA DE BIBLIO TE CA RIOS DE 1923}

En las postrimerías del siglo XIX y al inicio del si glo XX los apo yos para la im plan ta ción de la CDU en Es pa ña fue ron he chos ais la dos; sin embargo, unos años mas tarde, esta trayec to ria abar ca rá a un nu me ro so gru po de bibliotecarios. La acción mas relevante que apo yó la im plan ta ción en Es pa ña de la CDU, ante la di so cia ción de los bi blio te ca rios es pa ño les, fue la Asam blea de 1923, de los miembros del Cuerpo Facultativo de Archiveros y bibliotecarios con el objeto de tra tar los problemas que apremiaban en las bibliotecas, que de bía ce le brar se los días 23 al 29 de oc tubre, pero tuvo que ser aplazadaal producirse el 13 de sep tiem bre de este mis mo año un golpe de es ta doencabeza do por Primode Rivera. El tema abor da do, de for ma prio ri ta ria, fue la implantación de un sistema de clasificación biblioteco-bibliográfica.Fue el primer intento en tre los miem bros del Cuer po fa cul ta ti vo de instrumen tar un sis te macla si ficatorio y en es pe cial la Cla si fi ca ción De ci mal para las biblio te cas y ca tá lo gos es pa ño les. Con la ce lebra ción de la Asam blea se tra ta ba de dar so lución a los problemas derivados de la organización de las biblio te cas públicas, también de la formación del catálogo central bibliográfico, y de los catálogos de materias. Pero este intento organizativo que abortado por el golpe de estado de Primo de Rivera ocurrido en 1923, y será durante la Segunda República española cuando se inicien, de nuevo, las gestiones en este sentido. La Asamblea hubiera podido fraguar y consolidar la implantación de la Clasificación Deci-

10 PAZ Y MELIA, Antonio. "La cuestión de las Bibliotecas Nacionales y su difusión de la cultura". En: REVISTA de Archivos, Bibliotecas y Museos, 1910 ; p. 109.

11 El Sistema de Brunet, o también denominada Clasificación de los libreros parisinos, fue el sistema difundido por el bibliógrafo y librero Jacques Charles Brunet durante el siglo XIX con la publicación de su gran repertorio bibliográfico Manuel du libraire et de l'amateur de livres. Debe su nombre a Brunet por ser éste quien difundió el sistema; a partir de la publicación del repertorio este esquema clasificatorio tuvo una gran difusión debido en gran manera, a la importancia del comercio del libro en París durante la pasada centuria. El sistema establece cinco grupos temáticos: A Teología; E Jurisprudencia; I Ciencias; O Artes y Bellas letras; U Historia.

12 PAZ Y MELIA, Antonio. Idem. (Cont.), año I, 1911.

13 MENDEZ ALB ARRAN, Luis. La Clasificación Bibliográfica Decimal, exposición del Sistema y de sus tablas compendiadas, 1931; p. 15. Luis Méndez Albarrán sacará a colación a Eguía como uno de los destacados difusores de la Clasificación Decimal en España, ya que en su obra Mi biblioteca. Clasificación, 1920; p.6-7, explicaba la clasificación bibliográfica rectora de su catálogo. Méndez Albarrán destacó también la diferencia expositiva de las tablas de Eguía respecto a las de Manuel Castillo debido a la acción remodeladora efectuada por el Instituto en las tablas de la Clasificación Decimal, puesto que las tablas de Castillo se habían quedado ya antiguas y obsoletas y, por el contrario, Eguía incluía las nuevas modificaciones. 
mal en las bibliotecas españolas, ya que fueron numerosos los bibliotecarios que pos tu la ron la im plan ta ción del Sis te ma De cimal a través de las comunicaciones que prepararon para la misma. Además, cabe destacar no sólo los fines que perseguía la convocatoria de la Asamblea, sino también los me dios para su con vo ca to ria, por que ésta fue emitida mediante Real Orden.

En la Asam blea, al ini cio de su ce le bra ción, se tra tó de for ma te nue la proble má ti ca de un sistema clasificatorio, aunque algunos mostraron ma yor in te rés al res pec to como fue ron Ignacio Rubio y Cambronero, José de San Simón, José María Castrillo, Jesús Domínguez Bordona y Rafael Ureña. El bibliotecario vallisoletano Ignacio Rubio y Cambronero mostró gran sensibilidad por los problemas técnicos de las bibliotecas, publican do un artícu lo en la me mo ria ge ne ral de la Asam blea del Cuer po de Ar chi ve ros y Bi bliote ca rios bajo el tí tu lo "El li bre ac ce so a los estantes en las bibliotecas del Estado", ${ }^{14}$ en el que postulaba la necesaria implantación de unaclasificaciónbibliográficaqueposibilitara la normalización en materia de clasificación do cu men tal, y en es pe cial plan teó la posibilidad de adopción de la Clasificación Decimal. Su de fen sa del Sis te ma De ci mal se vio acompañada de las propuestas, en este mismo sentido, de otros biblio te carios.

José Ma ría Cas trillo es tu vo des ti na do como bi blio te ca rio en la Fa cul tad de Fi lo so fía y Letras de la Universidad Complutense de Madrid. Expuso, al igual que otros bibliotecarios, una ponencia en la Asamblea titulada "Catálogo por materias", en la que ponía de manifies to la ne ce si dad de adop tar un cua dro de clasificación bibliográfica para clasificar las bibliotecas españolas. ${ }^{15}$ Ante este vacío pro pu so que en la ci ta da Asamblea se di ri miera y votara la implantación de un sistema y éste pu die ra ser el De ci mal, ya que, se gún su criterio, se había llevado a la práctica. No dudó en ins tar a sus com pa ñe ros para lo grar la implantación de un cuadro clasificatorio.
Jesús Domínguez Bordona, al igual que José Ma. Cas tri llo, hizo alu sión, en la Asamblea, a la au sen cia de un es que ma cla si fica torio biblioteco-bibliográfico que debía haberse incluido en las Instrucciones para ca tálogos en las Biblio te cas Públicas de 1902.

${ }^{16}$ En la Asam blea, ce le bra da vein te años después de la emisión de las Instrucciones, se puso de manifiesto esta gran carencia de las mis mas y la ur gen te ne ce si dad de adap tar un sis te ma cla sifica to rio uni for me para to das las bibliotecas estatales. También existieron voces que se alzaron desde el ámbito de otros gru pos profe siona les como ocu rrie ra con $\mathbf{R} \mathbf{a}$ fael UreñaSmen jaud que, sien do ju ris ta catedrático de Historia del Derecho, llevó a cabo una apli ca ción prác ti ca del Sis te ma Deci mal en labiblio te ca de su "la bo ra to rio", que comprendía 30.000 volúmenes. De hechos como éste se desprende la gran sensibilidad ante los proble mas bibliográficos que mos traron los juristas, pues este grupo profesional ha ex pre sa do su in quie tud y de di ca ción a esta te mática, a sa ber: eran ju ris tas Otlet y La Fontaine. En España, además de destacar numerosos bi blió grafos, cabe men cio nar a Mo re no Nie to, To rres Cam pos, Co dor niú, en tre otros, que pro pug na ron un gran avan ce en las téc nicas bibliográficas y por ende en las cla si ficatorias.

El último gran propulsor de la CDU en la Asam blea fue José de San Si món, que te nía a su cargo la Bibliote ca del Jardín Botánicoen cuya clasificación adoptó, por decisión propia, el Sistema Decimal. Con anterioridad, ha bía em plea do una guía cla si fi ca to ria que él mismo había hecho, pero resultó ser un plan arbitrario e ineficaz para organizar el catálogo. Des ca li fi có este in ten to e im plan tó la Clasi fi ca ción De ci mal. Abor dó una gran de fen sa del sis te ma De ci mal en la Asam blea de 1923. ${ }^{17}$ En de fi nitiva, ve mos que im plan tó la Cla sificación Decimal en la Biblioteca del Jardín Botánico para organizar los catálogos y también los fondos. Este esquema clasificatorio abarcaba casi la to ta li dad de los fon dos de la biblio te cacuan doque dó in te rrum pidaestalabor a causa de la Guerra Civil española y la muerte de San simón, acaecida en 1935 o 1936. ${ }^{18}$
Todas estas iniciativas se vieron truncadas con el gol pe de Es ta do de 1923, ya que la acti vidad de la Asamblea se vio in te rrum pi day con ella el intento, por parte de numerosos bibliotecarios, de aceptar el sistema clasificatorio que propagabael Insti tu to de Bruselas. Trasel in ten to fraca sa do de la Asamblea, Camilo Chousa fue el pri me ro que, de nuevo, trató de difundir el Sistema Decimal. DestacaChousaen tre aquellos profesionales no bibliotecarios que abordaron problemas bibliográficos y bibliotecarios. En 1927 publicó una obra en la que hacía un análisis de los sis te mas cla si fi ca to rios, bajo el tí tu lo Biblioteconomía. Sistemas de clasificación. Hizo una so me ra ex po si ción de las cla si ficaciones que habían tenido una aplicación bibliográfica práctica, defendiendo la Clasificación Decimal. ${ }^{19}$

\section{INCIDENCIA DEL SISTEMA DECI- MAL EN CATALUÑA}

El Sis te ma de ci mal es tu vo su je to a una evo lut ción his tórica di fe ren te en Ca ta lu ña, se gún ya hemos reseñado al ha cer men ción de la ta rea emprendida por el bibliotecario Sebastián Far nés. Ya que en Ca ta lu ña na ció una or ga nización biblio tecariapropiamentecata la nasin depender de la Administración central sino, del go biernoau tó no mo de la Man co munidad de Ca ta lu ña. Su gé ne sis vino de ter mi na da por varios fac to res, en tre los que des ta ca la existen cia de un pro le ta ria do fa bril y el obre ro industrial — con mayor nivel educativo que el cam pe sinorural-y am bos gru pos pre sionaron para lograr un mayor acceso a la cultura. Tam bién ori gi nó la crea ción de esta or ga ni zación bibliotecaria catalana el acrecentamiento del mo vi mien to na cio na lis ta ca ta lán a fina les del siglo XIX. La creación en 1914 de un go bier no con cier to gra do de au to no mía, respecto al gobierno central, también posibilitó este de sa rro llo bi blio te ca rio de ám bi to nacional. El Presidente de la Mancomunidad de Cataluña Prat de la Riba gestionó esta creación y con tó con la re le van te co laboración del biblio te cario Jor di Ru biói Ba la guer. ${ }^{20} \mathrm{Ru}$ bió ejerció una gran actividad bibliotecaria en Ca ta lu ña, ya que en 1914, al crear se la Bi blio-

14 RUBIO Y CAMBRONERO, Ignacio."El libre acceso a los estantes en las bibliotecas del Estado”. En: REVISTA de Archivos, Bibliotecas y Museos, 1923.

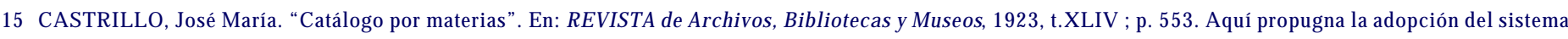

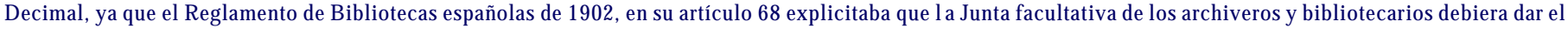

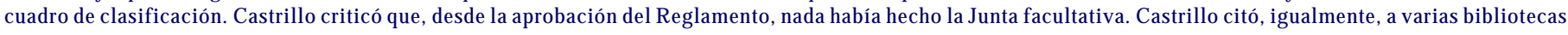
españolas que habían aplicado el Sistema Decimal como la Biblioteca de Arquitectura, el Centro de Estudios Históricos, Jardín Botánico e Ingenieros del Ejército. La

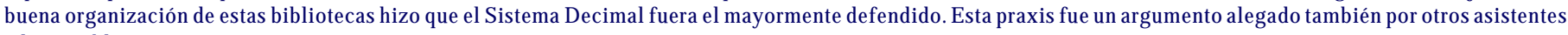
a la asamblea.

16 Aprobadas mediante Orden de 31 de julio de 1902, y presentadas por la Junta Técnica de Archivos, Bibliotecas y Museos.

17 SAN SIMON, José de. "Los catálogos de las bibliotecas". En: REVISTA de Archivos, Bibliotecas y Museos, 1923, XXVIII ; p. 554.

18 Según Francisco Rocher, que en 1965 ocupaba la plaza vacante de San Simón.

19 CHOUSA, Camilo. Biblioteconomía, sistemas de clasificación, 1927.

20 FAUS SEVILlA, Pilar. La lectura pública en España y el Plan de Bibliotecas de María Moliner; p. 47. 
te ca de Ca ta lu ña, ocu pó la di rec ción de ésta y, posterior men te, im partió do cen cia de Biblioteconomía, en 1915, enseñando téc ni cas clasificatorias en la Escuela de Bibliotecarias. Tam bién fue di rec tor de la Red de Bi blio te cas Populares fundada durante el gobierno de la Mancomunidad de Cataluña. Vemos, pues, que con la crea ción de la Es cue la de Bi blio teca rias, la Biblio te ca de Ca ta lu ña y la red de biblio te cas po pu la res se ges tó una or ga ni za ción bibliotecaria sólidamente construida y única en el territorioespañol. ${ }^{21}$ Esta situación propi cióla im plan tación del Sis te ma De cimalen toda la extensión bibliotecaria y biblioteconómica catalana. Cabe aña dir a este res pec to que, con anterioridad a la creación de la Biblioteca de Ca ta lu ña, en 1912, se ha bía de cidi do, ade más, que ésta se or ga ni za ra se gún el SistemaDecimal, ${ }^{22}$ y por ende en la red de bibliotecas de Cataluña. Por estos hechos, en la Escuela de Bibliotecarias se impartieron enseñanzas que colaboraron a difundir el Sistema Decimal.

Jordi Rubió fue el gran promotor, difusor y adap ta dor de la Cla si fi ca ción De ci mal en Cata luña. Ante es tas iniciativas, al co mien zo del si glo XX se ini ció en Ca ta lu ña una políti ca bi bliotecaria similar a la que tenían los países más desarrollados de Europa, y la adopción del Sistema Decimal fue uno de sus pilares. Ru bió fue un des ta ca do es tu dio so de la Clasificación De ci mal, pero su re le van cia no radicó sólo en ser un teó ri co y tra duc tor del Sis tema Decimal, sino que también logró la implantación del Sis te ma en la Bi blio te ca de Cataluña y en las bibliotecaspopulares de la Mancomunidad, en tanto que director de éstas. Pero ade más cen tró su de di ca ción bi bliotecariacomoCatedráticodeBiblioteconomía en la Escole Superior de Bibliotecaries de la Mancomunidad, dondetrabajó como investiga dor y di fu sor de la Cla si fi ca ción De ci mal., Comenzó pronto su labor teórica. En 1920 publicó su primera y más destacada traducción de las tablas bajo el título Clasificació De cimal, adap ta ció pera les biblio te ques catalans. Esta traducción se basó en la versión del Ins ti tu to de $1905^{23}$ titulada Ma nuel du répertoirebibliographiqueuniversel. Jor di Rubió rea li zó esta tra duc ción, ya que por acuerdo del Instituto de Estudios Catalanes, el Sis te ma Deci mal se implan tó en la Biblio teca de Cataluña (la primera gran biblioteca del Estado español que lo hizo), y como las bibliotecas populares catalanas eransucursales de la Biblioteca de Cataluña, y entre ellas se posibilitaba el préstamo de libros y acceso a todos los catálogos, ello supuso que, igualmente, se implantara el Sistema Decimal en las ci ta das bi blio te cas po pu la res. Así pues, la tra duc ción de Ru bió fue la base teó ri ca y meto do ló gi ca para la or ga ni za ción de los ca tálogos en todas las bibliotecascatalanas.

Ru bió se apar tó de la or to do xia y mo di fi có el nú me ro asig na do en las ta blas a la len gua cata la na, ya que ésta apa re cía como dia lec to del francés (modificación y distanciamiento que había puesto de manifiesto Sebastián Farnés en la Bi blio te ca del Fo men to del Traba jo Na cional, según ya señalamos). La adaptación catalanadel ManuelduRepertoire BibliographiqueUniversel, he cha por Rubió, con tó con la autorización del Instituto Internacional de Bibliografía. Esta adaptación fue objeto de críticas, como las manifestadas por el bibliotecario Javier Lasso de la Vega ${ }^{24}$ En 1938 Rubió pu bli ca una se gun da edi ción de su traducción y adaptación de las tablas, y tomó esta segunda versión de la Clasificación Deci mal de 1905, al igual que la pri me ra. Ru bió prefirió optar por la línea primera de actuación del Ins ti tu to, ya que en las pri me ras conferenciasbibliográficas de ámbitoin ternacional, se había defendido el principio de invariabilidad de las tablasclasifica to riasrespecto a las tablas de Dewey. Pero en la decimo se gun da con fe ren cia la po si ción del Ins tituto tendió a modificar las tablas. Y, según Ru bió, es en este mo men to cuan do se produce una mayor diferenciación entre las tablas de la CDU y el sis te ma de ci mal. Las ta blas de la CDU, a partir de este mo men to, van a tra tar de expresar con mayor cantidad de símbolos y exactitud las infinitas modulaciones de los con te nidos y te máticas de los libros, mien tras que el Sis te ma De ci mal de Dewey se man tenía fiel al prin ci pio de cla ri dad y sim pli ci dad. O sea, cuan do Ru bió pu bli ca su se gun da edición ya se había producido el nacimiento, propiamente dicho, de las tablas de la CDU, en su se gub daedi ción in ter na cio nal de las tablas (1927-32). A pe sar de ello Ru bió no dudó en considerar que toda clasificación es un mero convencionalismo y que, además, esta nue va edi ción de las ta blas de la CDU pre sentaba numerosos inconvenientes, ya que perdía los criterios de claridad y simplificación que Dewey había defendido, porque aumentaba notablemente el número de divisiones sistemáticas frente a la primera versión de 1905.

Ru bio se apo yó tam bién en mu chos otros argumentos en su defensa de la Clasificación De ci mal, fren te a la CDU. Para ello ar gu mentó que, aun que la apa ri ción de la CDU mar có un período de gran difusión, ello no supuso que se adoptara de forma ortodoxa como suce dió en la URSS, Rei no Uni do, y otros países. Otro de los ar gu men tos en los que se apoyó fue con si de rar que, ya en 1938, la CDU no tenía la importancia an te rior, como se vio en la reunión del Instituto Internacional de Documentación (nombre que tomó el Instituto de Bruselas a partir de 1931) que tuvo lugar en Fran kfurt en 1932, en la que el pre si den te destacó que en tre las más im por tan tes mi siones del IID no figuraba la de hacer valer la CDU. Y tam bién en la re u nión de la Ame ri can Library Association (ALA) se concluyó que la CDU no era un sistema bibliotecario sino más bien un sis te ma re serva do a las bi blio gra fías, es de cir, la CDU era un len gua je para designar conceptos no para ordenarlos. ${ }^{25}$ Por todo ello, Rubió no empleó la CDU sino el sistema que la había originado: la clasificación De ci mal de Dewey con las pri me ras modificacionesque hizo el Ins ti tu to en 1905.

Ru bió tra ba jó por el de sa rro llo teóri co y práctico de la Clasificación Decimal hasta que a causa del golpe de estado de 1923 fue destituido de sus cargos, aunque con el posterior as cen so de la Se gun da Re públicaes pañolade nue vo re gre sa a su car go has ta 1939, mo mento en que finaliza la Guerra Ci vil Es pañola.El 26 de enero de 1939 Barcelona fue ocupada por las fuer zas fran quis tas, don de lle gó un comi sario de biblio te ca del Go bierno de Burgos (o también denominado Gobierno del bando nacio nalis ta) que descalificóla la bor "ca tala nis ta de Ru bió”. Días des pués se hizo la des titución formal de Rubió de la dirección de la Biblio te ca de Ca ta luñay de su cargo docente. Destituído Rubió no prosiguió trabajando para el desarrollo de la

21 FAUS SEVILLA, Pilar. Idem.; p. 18.; p. 48.

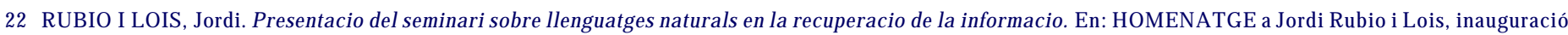
del curs academic, 1988-1989; p.18-19.

23 RUBIO I BALAGUER, Jordi. Classificació decimal, adaptacio per a les biblioteques catalanes. Próleg a l'edició de 1920 ; p. VII.

24 LASSO DE LA VEGA, Javier. La verdadera historia de la Clasificación Decimal de Dewey.

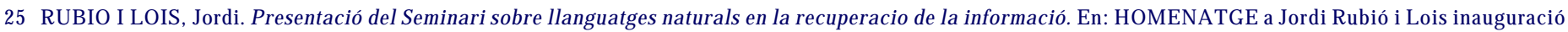
del cours academic, 1988-1989, op. cit.; p. 20. 
Clasificación Decimal hasta 1976, año en el que publicó la tercera edición de su adaptación de la C.D. La cuar ta ver sión de esta adapta ción ca ta la na fue he cha por su hijo Jor di Rubió i Lois. Jor di Ru bió con tri bu yó a im plan tar la Clasificación Decimal en las bibliotecas catalanas, tradujo las tablas e hizo diversos es tu dios en los que ex po nía una bre ve di ser tación sobre la teoría de la Clasificación, los distintos sistemas y un pequeño desarrollo explicativo del Sistema Decimal; entre estas obras des ta can: Cómo se or ga niza y ca ta lo ga una biblioteca, Catalogación y ordenación de bibliotecas, Instrucciones documentales, Libros y bibliotecas, una car ti lla para suordenación. ${ }^{26}$ Esta só li da apo ya tu ra teó ri ca sut puso que, como director de la Biblioteca de Cataluña, emprendiera novedadesrevolucionarias en la mis ma, como do tar a la Biblio te ca de una par te de los fon dos de ac ce so di rec to y otra par te para prés ta mos, ade más de adap tar la Cla si fi ca ción De ci mal no sólo para el ca tá logo sistemático de materias, sino además para la ordenación de los libros de la sala de accesodirecto y tam bién los li bros de los depósitos, realidad que únicamente acontecerá en Cataluña, ya que en el resto de España la clasificación va a es tar su je ta a otros fac to res.

\section{IMPLANTACION DE LA CDU EN EL MARCO DE LA SEGUNDA REPU- BLICA ESPAÑOLA}

El 14 de abril de 1931 se pro cla mó la II Re pública española, el período de la Restauración fi na li zó y se ini ció un mo men to de gran preocupación por las bibliotecas, en el que se abor da ron los proble mas ca pi ta les delámbi to bibliotecario, entre ellos: la clasificación. Pilar Faus des tacalare le van cia de este pe ríodo cuando expone: "No cabe duda que estamos en presencia de uno de los momentos más efervescentes y positivos de la historia de nuestras bibliotecas públicas". ${ }^{27}$ Durante el período de la República se articuló un gran de sa rrollo de la políti ca bi blio te ca ria y se implan tó la Cla si fi ca ción De ci mal en la Bi blioteca Nacional, en las bibliotecas populares, en las uni versi ta rias, en las mu ni ci pa les, y en otras, aun que no se dotó a es tas ex pe rien cias iniciales de una base le gal para evi tar la ri gidez y constreñimiento legalista, y así poder in troducirmodificaciones se gúnel dictadode la experiencia. Sin embargo, hubo quienes criticaron esta flexibilidad y apuntaron que erapreferibleop tar porconsolidarlegalmente todo cuan to se ini cia ra, así lo se ña la ban el joven bibliotecario facultativo Javier Lasso de la Vega, quien fi na li za da la gue rra pro mo vió una gran la bor, en este sen ti do, fun da men talmente legislativa.

La República inició pronto el desarrollo de una política bibliotecaria, ${ }^{28}$ recogiendo las ideas edu ca ti vas más avan za das; se pre ten dió el fo men to de la lec tu ra me dian te el es table cimien to de bi blio te cas po pu la res fi jas y circulantes, mu nicipa les y otras, to das ellas atravesadas por el tamiz de las ideas de la Institución Libre de Enseñanza y las ideas progresistas emanadas por otros países. Es así, en ton ces, por lo que la crea ción, por par te del gobierno republicano, de varias instituciones aledañas al libro y las bibliotecas, como fueran el Patronato de Misiones Pedagógicas, de la Jun ta de In ter cam bio y Ad quisición de Libros para Bibliotecas Públicas, delCon se jo Cen tral de Archi vos, Biblio te cas y Te so ro Ar tís ti co y de la Sec ción de Biblio tecas de Cultura Popular, nosupusosolamente la asun ción de las ideas más pro gre sis tas en o que hace referencia a una organización bibliotecaria sino también en lo que respecta a los trabajos técnicos.

Así, la Cla si fi ca ción De ci mal se hizo ex ten siva a to dos los ám bi tos bi blio te ca rios. Las instituciones emanadas de los distintos órganos directivos apuntaban a su totalimplantación. La creación, mediante Decreto de 21 de noviem bre de 1931, de la Jun ta de In ter cam bio y Ad qui si ción de Li bros para bi blio te cas públicas contó con la colaboración de numerosos bibliotecarios facultativos que pretendieron modificar las bibliotecas españolas. Esta colaboración estuvo dirigida hacia la selección de libros actuales, a la ayuda a los lectores menos formados y al empleo de modernas técnicas bibliotecarias. La Junta nació para modernizar las colecciones bibliográficas, aunque, debido a la situación precaria del país, su mi sión prio ri ta ria se tor nó en la creación de bibliotecas, y en 1932, mediante decre to del 13 de ju lio, se crea ron las nue vas bibliotecas municipales. Además, la Junta re dac tó un re gla men to de és tas en el que consta ba el ré gi men y servi cios de lec tu ra y prés tamo y en vió va rias cir cu la res a los en car ga dos de las biblio te cas munici pa les con ins truc ciones para la or ga ni za ción de las bi blio te cas. En estas ins truc cio nes se ha cía ya re fe ren cia a la Clasificación Decimal.

Du ran te la Gue rra Ci viles pa ñola de 1936- 39 la dis po si ción más im por tan te, en este sen tido, por la par te del go bier no re publica no, fue la crea ción del Con se jo Cen tral de Ar chi vos, Biblio te cas y Tesoro Artístico, me dian te Decre to de 16 de fe bre ro de 1937 , las atri bu ciones del Con se jo abarcaban to das las re fe rentes al tesoro artístico y documental, además de dic tar las ins truc cio nes y nor mas rec to ras de las bibliotecas y otros centros. La Presiden cia del mis moreca yó en To más Na va rro; la Subdirección de Bibliotecas Científicas, en Be ni to Sán chez Alon so; la Sub sec ción de Bibliotecas Ge ne ra les, en Juan Vi cens de la Lla ve; la de Biblio te cas Es co la res, en Ma ría Moliner; y la de Extensión Bibliotecaria,en Teresa de Andrés. Todos ellos estuvieron en car ga dos de dic tar las ins truc cio nes y normas para la ejecución de los trabajos técnicos en las bi blio te cas, ya que ésta era una de las atri bu cio nes del Con se jo, se gún la Orden de 5 de abril de 1937, y en tre los tra ba jos técnicos figuraba la clasificación temática, tarea que fue abordada por los citados miembros del Consejo.

Tomás Navarro Tomás también ocupó el car go de Se cre ta rio de la Sub sec ción de Bibliotecas His tó ri cas, ade más de la pre si dencia del Con se jo; fue di rec tor de la bi blio te ca Nacional y un gran defensor de la CDU. Supo apoyarse en sus compañeros del Conse jo que igual men te se ocu pa ron con des velo de las directrices de los trabajos técnicosen las bibliotecas.

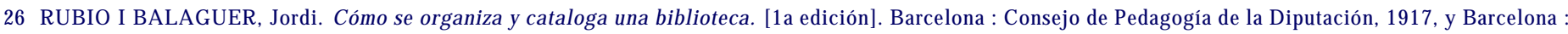
Cámara Oficial del Libro, 1932. RUBIO I BALAGUER, Jordi. Catalogación y ordenación de bibliotecas. Instrucciones elementales. Barcelona: Tabor, [1928?] y Barcelona: W-A-L, 1946. RUBIO I BALAGUER, Jordi. Libros y bibliotecas, una cartilla para su ordenación. Barcelona : Gremio de Editores y Librerías, 1952.

27 FAUS SEVILlA, Pilar. La lectura pública en España y el Plan de Bibliotecas de María Moliner, op. cit.; p. 61.

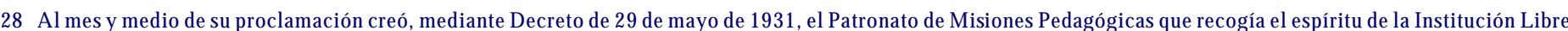

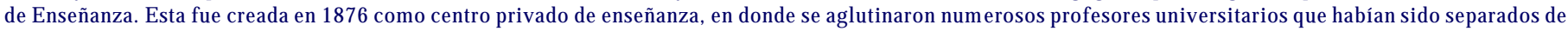
sus cátedras por su ideas liberales, dirigida por Francisco Giner de los Ríos, y a su muerte por Manuel Bartolomé Cossío. A la Institución Libre de Enseñanza se debió también la creación de la Junta de Ampliación de Estudios en 1907. El Presidente de la Junta fue Santiago Ramón y Cajal, investigador que ya contaba con un prestigio reconocido (en 1906 obtuvo el Premio Nobel). Formaron parte de la Junta numerosos institucionistasque recogieron las ideas de Giner de los Ríos y trabajaron para que la preparación de los jóvenes investigadores se hiciera en centros prestigiosos extranjeros. También pretendieron agrupar a los estudiosos y crear centros para ello dependientes de la Junta, y en 1910, a instancia del Ministro de Instrucción Pública, el conde de R omanones, se creó el Centro de Estudios Históricos, en donde se formó una biblioteca de estudios históricos pionera, no sólo por ser el primer centro que creó la Junta y por albergar en su génesis las ideas más novedosas de renovación científica, sino porque su biblioteca fue modélica al ser organizada mediante el Sistema Decimal. Además, cabe subrayar que el Ministerio de Instrucción Pública de la

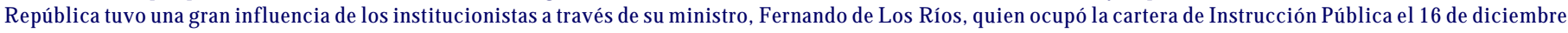

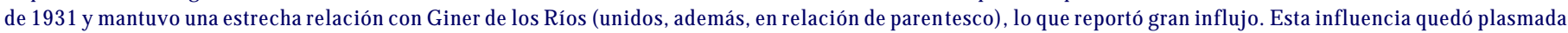
en todas las acciones que, en esta dirección, emprendió la República. 
El secretario del Consejo, Benito Sánchez Alonso, des ta có por ser his to ria dor y des tacado especialista español en historiografía, colaborador del Cen tro de Es tu dios His tóricos y también miembro del Cuerpo Facultativo. Con la crea ción de la Jun ta de In ter cam bio y Ad qui si ción de Li bros para Bi blio te cas públi cas ac ce dió a la pre si den cia de ésta en re presen tación de la Jun ta Fa cul ta ti va; ade más fue colaborador del Centro de Estudios Históricos y co no cía muy bien la Cla si fi ca ción De cimal, fue pio ne ro en la di fu sión de la mis ma. ${ }^{29}$ Defendió el Sistema Decimal observando la necesidad de traducir las tablas generales com ple tas y no abre via das, ya que la es pe cialización de numerosasbibliotecas lelle varon a considerar la traducción de Castillo insuficiente e inválida para aplicarla a grandes biblio te cas (la tra duc ción de Cas til lo ca re cía de las subdivisiones completas y detalladas de cada nú me ro de las ta blas de la CDU). Por el contrario, se gún ad ver tía Sán chez Alon so, el Servicio de Información de la Biblioteca de In ge nie ros delEjér ci to ha bía ela bo ra do la tra duc ción de las ta blas re la ti vas al nú me ro 62 y pudo por ello aplicarse el Sistema Decimal con la pertinencia exigida en una biblioteca tan especializada.

Vicens de la Llave, que tenía a su cargo la Sub sec ción de bi blio te cas ge ne ra les del Consejo, fue miembro del Cuerpo Facultativo y ocu pó el car go de Ins pec tor de las bi blio te cas municipales de Ma drid. ${ }^{30}$ Participó activamente en el desarrollo y creación de las bibliotecas de las Misiones Pedagógicas y trabajó en la Oficina Técnica de la Junta de Intercambio junto con otros tres bibliotecarios. La Ofi ci na Téc ni ca pre ten dió el em pleo de modernas técnicas bibliotecarias y, por tanto, de los sistemas clasificatorios. Vicens de la Llave abogó por la implantación de un sis te ma cla si fi ca to rio ade cua do. Así, en 1934 publicó un artículo bajo el título: "Catalogación y cla si fi ca ción”, don de ex pu so la ne ce si dad de una mejora organizativa, de carácter ofi cial, de las bi blio te cas del Es ta do en lo que se refería a la clasificación sistemática. Ya que exis tían ins truc cio nes ofi cia les para la ca talogación alfabética, pero nada existía ni se había hecho en lo que hacía referencia a la Clasificación Sistemática. ${ }^{31}$ Vicens de la
Llave consideró que esta problemáticadebía re sol ver se con la adop ción de un sis te ma cla sificatorio, por lo que promovió la implantación de la CDU y fue un entusiasta defensor. Mas finalizada la Guerra civil se exilió en México donde publicó: Cómo se organiza unabiblioteca y Manualdelcatálogodiccionario, pu bli ca das am bas en 1942; ade más publi có en Pa rís, en 1938, L'Espagnevivante: le pueple à la Conquête de la Culture, esta última es una gran apor ta ción en el ám bi to bi bliote cario es pa ñol y casi por com ple to des conoci daen Es pa ña.

María Moliner, que tenía a su cargo la Subsección de Bibliotecas EscolaresdelConsejo, erabiblio te ca ria miem bro delCuer po Facul ta tivo; ayudada por otros bibliotecarios realizó un plan de bibliotecas públicas, que apareció como una publica ción del Con se jo Cen tral de Archivos, Bi blio te cas y Te so ro Artís ti co bajo el tí tu lo: Plan para una organización general delas Bibliotecas Públicas. Ha sido el primer plan que diseñaba la organización bibliotecaria es pa ño la con ce bi da como una uni dad, o lo que es lo mis mo, un sis te ma bi blio te ca rio propia men te di cho. En el plan fi gu raban ór ga nos centrales en los que quedaba incluida la Sección de BibliotecasdelConsejo, encargadade las directrices de las tareas técnicas y, por ende, del sis te ma cla si fi ca to rio rec tor de la organización de catálogos, bibliografías y fondosbibliográficos. La Oficina de Adquisición de Libros también tendría encomendada de forma colateral esta tarea en tanto que era la encargada de la catalogación, al igual que la Oficina del Catálogo General, que elaboraría un catálogo colectivo general de todas las bibliotecas públicas españolas.

Como miembro del Consejo, destaca de forma muy especial María Moliner, autora del conocido Diccionario del uso español al que se dedicó,finalizada la Guerra Civil, cuando fue relegada de su intensaactividadbiblioteca ria. Du ran te la Gue rra Ci vil ocu pó el car go de Jefe de la Biblioteca Universitaria y Provincial de Valencia. A finales del año 1937 abandonó la direc ción para de di car se, de forma com ple ta, a su otra ta rea di ri gien do la Oficina de Adquisición y Cambio Internacional de Pu bli ca cio nes y tam bién como vo cal de la Sección de Bibliotecas del Consejo Central de Archivos, Bibliotecas y Tesoro Artístico. Es tas dos ins ti tu ciones se en car ga ban de toda la políticabilio te caria. Des de esta posiciónse publicaron dos importantísimas aportaciones: Pro yec to de ba ses de un plan de or ga nización general de bibliotecas del Estado, en 1939, e Instrucciones para el servicio de pequeñasbibliotecas, en 1937, son las pri me ras ins trucciones es pa ñolas que pres criben elempleo de la CDU; se dirigieron a pequeñas biblio te cas pues el go bier nore publica nocen tró su aten ción en las mis mas. Es tos dos obras resumieron la intensa actividad cultural y bibliotecaria desarrollada en los años de la República (1931-1939), aunque en los años correspondientes a la Guerra Civil esta actividad se in ten si ficó so bre mane ra, y se pro du jo una eclosión de actividades culturales y bibliotecarias. Este período tan fructífero fue un renacimiento cul tu ral es pa ñol co no ci do como la Edad de Pla ta de la cultura española. ${ }^{32}$ María Moliner, en su car go como vo cal de la Sec ción de Biblio te cas del Con se jo Cen tral de Ar chi vos, Biblio te cas y Te soro Ar tís ti co, hizo las ci ta das ins truc cio nes que son de gran im por tan cia por su ori gi na li dad, y por tratar se de las pri me ras nor mas de este tipo. En ellas se plas mó la im plan ta ción de la Cla si fi ca ción Decimal. ${ }^{33}$

Teresa de Andrés Zamora tuvo a su cargo la Extensión bibliotecariadelCon se jo, colaboróinten sa men te con Cul tu ra Po pu lar y ela bo ró para la misma las Indicaciones para la organización de frentes, cuarteles y hospitales, que guardan una estrecha concomitancia con las Instrucciones parael serviciode pequeñas bibliote cas que María Moliner redactó. El vínculo entre ambas instruc cio nes es gran de, pero aquí cabe des ta car que éste es tribaen que am bas bi blio te ca rias ocu pa ban car gos ale da ños en el Con se jo Cen tral de Archivos, biblio te cas y Te so ro Artís tico.

Resultó, pues, que todos los miembros que par ti ci pa ban en la di rec ción del Con se jo, órgano del que emanaban todas las instrucciones, eran abier tos par ti da rios de la im plan ta ción de la CDU en las bibliotecas españolas, como Sánchez Alonso, Vicens de la Llave, María Moliner, Teresa de Andrés y To más Na va rro. En las citadas Instrucciones se emitió la obliga to rie dad del em pleo de la CDU para la cla sificación de los fondos bibliográficos.

29 SANCHEZ ALONSO, Benito. "Sobre los índices impresos en las Bibliotecas públicas”. En: REVISTA de Archivos, Bibliotecas y Museos, 1915, año I ; p. 138-146. En este artículo propagaba la idea de implantar la Clasificación Decimal en las bibliotecas españolas. Advertía que este sistema clasificatorio estaría sujeto a las modificaciones realizadas por el Instituto. Sánchez Alonso consideró modélicos el Servicio de Información Bibliográfica de la Biblioteca de la Escuela de Ingenieros del Ejército, la Biblioteca de Arquitectura y la del Centro de Estudios Históricos, porque en ellos se había implantado el Sistema Decimal y en absoluto había resultado un fracaso.

30 FAUS SEVILLA, Pilar. La lectura pública en España y el Plan de Bibliotecas de María Moliner; p. 78. Aquí se pone de manifiesto que la inspección fue establecida por la Junta de Intercambio y Adquisición de Libros para Bibliotecas Públicas con el objeto de prestar ayuda y controlar las bibliotecas.

31 VICENS DE LA LLAVE, Juan. "Catalogación y Clasificación”. En: BOLETIN de Bibliotecas y Bibliografía, T. I, jul.-sept. 1934, no. 1 ; p. $24-25$.

32 FAUS SEVILLA, Pilar. Idem.; p. 88

33 ESPAÑA. Ministerio e Instrucción Pública. Consejo Central de Archivos. Bibliotecas y Tesoro Artístico. Sección de Bibliotecas. "Instrucciones para el Servicio de pequeñas Bibliotecas" / [por María Moliner]. En: LA LECTURA Pública en España. Op. cit.; p. 19. 


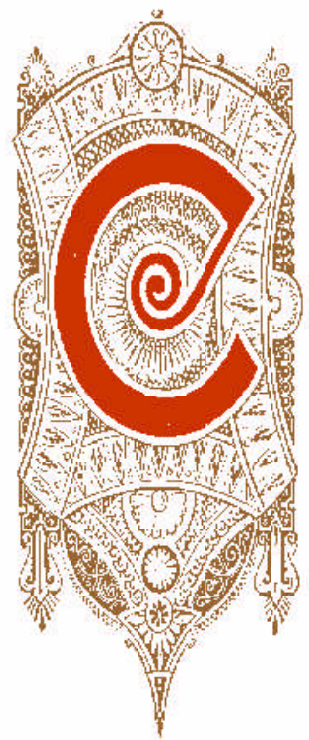

“... en las bibliotecas que pasaron a depender del Consejo a partir de 1937 se implantó la CDU , y lo mismo ocurrió en todas aquellas que el Patronato de las Misiones Pedagógicas había creado"

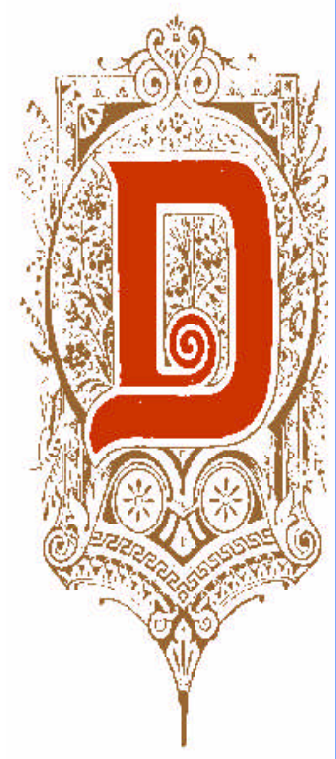

Así, en las bi blio te cas que pa sa ron a de pen der del Consejo a partir de 1937 se implantó la CDU, y lo mismo ocurrió en todas aquellas que el Pa trona to de las Mi siones Pedagógicas había creado.

Lanecesi dad de tra duc ción de las ta blas ge nera les de la Cla si fi ca ción De ci mal se hizo muy acuciante. El bibliotecario Méndez Albarrán ela bo róla pri me ra tra duc ción de ta lla da de las tablas ge ne rales, que con gran im pacien ciase esperaba. Mén dez Albarrán, biblio te cario del Centro de Estudios Extremeños, publicó un manual en 1932 sobre la Clasificación Decimal bajo el tí tu loLaClasificación BibliográficaDecimal. Exposicióndel siste mayde sus tablascompendiadas. En esta mo no gra fía incluyó, además, una traducción abreviada de las tablas para lo que hubo solicitado previa autorización del Ins ti tu to Internacionalde Biblio gra fía de Bru se las, y que fue in clui da dentro de las pu bli ca cio nes de ca rác ter ofi cial del Ins ti tu to con el nú me ro 167. La obra de Méndez Al ba rrán tuvo una gran di fu sión en Es paña, pues el sis te ma De ci mal ya te nía bas tan te vi gen cia y ha bía sido im plan ta do en nu me rosas bibliotecas. Además, las tablas aparecieron expuestas de forma sencilla y clara; esto convirtió al manual en un apoyo práctico y útil para los biblio te carios es pañoles. ${ }^{34}$ Méndez Albarrán se nos presenta también como teó ri co de la Cla si fi ca ción Bi blio gráfíca. Es ta ble ció una cla ra di fe ren cia ción en tre la sistematización exclusivamente científica y la Clasificación Bibliográfica. Se pronunciópor la ne ce si dad de rea li zar un es tu dio del sis te ma elegido seguido de una práctica consuetudina ria, es de cir, de li mi tar la cla si fi ca ción de las ciencias de la catalogación documental y del proceso transformador de aquélla en instrumento bibliográfico. ${ }^{35}$ Asimismo, trató de ex po ner una teo ría del Sis te ma De ci mal y reco no ció a Ma nuel Cas til lo, Ju lián de Eguía y Leo pol do Ji ménez como di fu so res en Es pa ña del Sis te ma De cimal, omitien do otras aportaciones y trabajos no tables como los ex pues tos anteriormente.

Vemos, pues, que la Clasificación decimal durante el período de la II República Españolaque dódefinitivamenteimplantadaeincu so para pe que ñas bi blio te cas, que eran las biblio te cas mas nu merosas. Por el con trario, la traducción de las tablas empleadas en bi- bliotecas mayores era la vertida por Méndez Albarrán.

Aho rabien, Ma ría Moli ner, que te nía gran conocimiento de la situaciónbibliotecaria y biblioteconómica española, remite en sus instrucciones a los bibliotecarios que deseen trabajar con mayor profundidad a la traducción de Méndez Albarrán. La Sección de bibliotecas trabajó en el desarrollo y creación de nu me ro sas biblio te cas en las que la Cla si ficación Decimal quedó definitivamente implantada. Aunque no se dio a esta implantación forma legal (ni tampoco a las ins truc ciones de 1937, ni las Ba ses de un plan de or ga ni za ción ge ne ral de bi blio te cas delEstado), porque se optó por estudiar, primeramente, la problemática que surgiera de estas ini cia ti vas, y se tra tó de evi tar la ri gi dez de la asunción legal de estos proyectos. De esta forma se posibilitaba introducir reformas y modificaciones.

Esta po líticabiblio te ca ria tan fruc tífe ra re nunció a una apoyatura e inmersión en el estricto mar co le gal. No se le gis ló so bre nor mas re la tivas a las ta reas téc ni cas, aun que esta ac ti vi dad se abor dó en el seno de los or ga nis mos res ponsales de la política bibliotecaria como el Ministerio de Instrucción Pública, el Consejo Cen tral de Ar chi vos, Bi blio te cas y Te so ro Artís ti co y la Ofi ci na de Ad qui si ción de Li bros y Cambio Internacional. Todos ellosubicados a partir de 1937 en Va len cia, por que el Go bier no de la Re pú bli ca du ran te la Gue rra Ci vil tras ladó su eje po lí ti co e in te lec tual de Ma drid a Valen cia. Allí, ade más, se ins ta ló un gran nú me ro de profesores universitarios e intelectuales.

Va len cia se con vir tió en la ca pi tal cul tu ral de España, y durante este período pequeño de tiem po vi vió una eclo sión de ac ti vi da des cultu ra les y tam bién bi blio te ca rias. Es tas ac ti vidades cobraron su latencia en una praxis y aun que ema na das de or ga nis mos ofi cia les no lograron cobertura legal. Derrotado el Gobier no de la Re pú bli ca por las tro pas de Franco y con clui da la con tien da, la asun ción de la Clasificación Decimal, dentro de un marco le gal, se va a pro du cir de ma nos del Go bier no insta la do en Vi toria.

\section{EMPLEO REGULADO DE LA CDU EN ESPAÑA}

La im plan ta ción de la CDU se va lió del ejercicio de la potestad reglamentaria del nuevo Gobierno y del Ministerio de Edu ca ción Nacio nal. Se im plan tó me dian te una Or den ministerial tres meses después de finalizar la Guerra Civil. Esta orden ministerial cubrió unalagunale gis la tiva decisivaen lo que hace

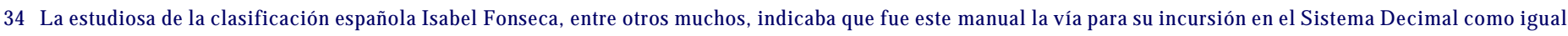
aconteciera a otras tantas promociones de bibliotecarios. FONSECA, Isabel. "La CDU en España". En: BOLETIN de la ANABAD, 1978 , año XVII, no.2. 
referenciaal de sarrollo de una po líticabibliote ca ria. La Or den se pro mul gó y en tró en vigor en 1939, sin embargo hacía alusión a un sistema clasificatorio cuya vigencia tenía ya casi una décaday es taba en con sonan ciacon las líneas téc ni cas eu ro peas. La pro yec ción de una política bibliotecaria hacia el exterior se ha bía ini cia do du ran te el pe río do de la Re pública. Un he cho significa ti vo, en este sen ti do, fue la ce le bra ción de 1935 del II Con gre so Internacional de Bibliotecas. Mediante este Congreso la política bibliotecaria española pretendió cobrar dimensiones fuera de nuestras fronteras, aunque esta apertura se vio obstacu lizada por los difíciles mo men tos que atravesaba España y Europa.

En España, tras ganar la guerra el bando nacionalista, se produjo un aislamiento que abar có tam bién el ám bi to bi blio te ca rio. En lo que respecta a Europa, la Segunda Guerra Mun dial obs ta cu li zó to das las la bo res y ac tividades del Instituto. Por ello, cuan do en España se es ta ble ció el em pleo de la CDU, este hecho no abarcó a las dimensiones internacionalistas que llevaba implícita la CDU, ni ob tu vo re so nan cia en el ex te rior. La im plicación de este hecho fue interior y sólo se dotó de un mar co le gal a una prác ti ca ya im pues ta. Esta apo ya tu ra le gal se pro du jo des de una línea ma yor men te teóri ca en la que se in ser ta ba Lasso de la Vega, quien pro mo vió y re dac tó la or den me dian te la cual se im plan ta la CDU en España.

Lasso de la Vega era bi blio te ca rio y miem bro del Cuerpo Facultativo desde 1915. Obtuvo una beca de unos me ses para es tu diar en Es tados Uni dos, lo que le con vir tió en in no va dor y teórico de las modernas técnicas bibliotecarias. Trabajó en la dirección de la Biblioteca Universitariade Ma drid y tam bién como docente de Biblioteconomía en la Facultad de Filosofía y Letras. Lasso de la Vega inició aquí una labor de propagación del Sistema Decimal, ya que, insertó en la cátedra de Bibliología, explicaba el uso y manejo de la CDU. Durante el Gobierno de la República par ti ci pó en la aso cia ción de Bi blio te ca rios y Bi blió gra fos de Es pa ña, en el II Con gre so Inter na cio nal de Biblio te cas y Biblio grafía donde co la bo ró como se cre ta rio del mis mo. Parti ci pó, tam bién, en la fun da ción y crea ción del
Boletín de Bibliotecas y Bibliografía. Antes del ini cio de la Gue rra Ci vil se mar chó a Portu gal, y du ran te elle van ta mien to de las tro pas de Fran co, el 18 de ju lio de 1936, se en con traba allí pró xi mo a par tir ha cia Es ta dos Uni dos. Regresó a España y trabajó en el bando nacionalista. Se incorporó a la Universidad de Se vi lla y más tar de tra tó de ex ten der a toda la zona na cio nal su la bor allí de sa rro lla da. Ocupó la Jefatura del Servicio Nacional de Biblio te cas y Archi vos, me dian te Orden mi niste rial de 26 de ene ro de 1938 , emi ti da du ran te la con tien da en el ladona ciona lis ta.

Desde la citada Jefatura realizó una fecunda laborlegislativa. ${ }^{36}$ La dis po si ción más acertada que promovió y redactó Lasso de la Vega, ya fue ra por su for ma ción o por su ac tividad bibliotecaria, fue el dar forma legal a una rea li dad en el ám bi to bi blio te ca rio es pañol: el em pleo re gu la do de la CDU. Pro movió y re dac tó la Or den de 29 de ju lio de 1939 mediante la cual se dictaminó que la organización de los fondos bibliográficos de las bibliotecas públicas se hiciera conforme al Sis te ma De ci mal de Mel vil Dewey y mo dificado por el Instituto Internacionalde Bibliografía.

Las ta blas com ple tas ge ne ra les de la CDU no es taban tra du ci das al cas te lla no, y se gún lacita da Orden és tas de bie ron ha ber se pu bli cado en los tres me ses si guien tes a par tir de la apa ri ción de esta Or den. La ver sión al cas te lla no de las tablas no se hizo hasta 1942, que fue realizada por Lasso de la Vega bajo el título: LaClasificación DecimalUniversal.Traducción abreviada precedida por una introducción so bre el con cep to y mi sión de bi blio te ca con una reseña de las principales clasificaciones y exposición del Sistema. ${ }^{37}$ Contiene una bre ve in tro duc ción que se apro xi ma a un breve tratado de la CDU, la traducción tuvo una gran acogida, ya que al implantarse la CDU, se hacía necesaria una traducción oficial de las ta blas. Sin em bar go, esta ver sión de las tablas fue autorizada por la FID, pero no estuvo incluida como publicación oficial de la misma. Y además tuvo como base la edición internacional fran ce sa de 1927-32, pese a que ya en 1938 se ha bía pu bli ca do la edi ción alemana. 38
En lo que respecta a la implantación oficial de la CDU ve mos que se pro mul go me dian te or den minis te rial en 1939 - jus to ter mina da la Guerra Civil- la cual obligó al uso de la CDU para la or de na ción de los fon dos de todas las biblio te cas públicas es paño las, se gún la edi ción de Ber lín, dice el De cre to, de bi do al entonces existente eje Berlín-Madrid. Ade más, como ve mos, la pro mul ga ción de la CDU comoclasi fica ción bi blio te ca ria en España es tu vo mar ca da por la im pron ta de asumir como base modélica la versión internacional alemana, debido a las estrechas relaciones mantenidas entre España y Alemania en este mo men to. Y en 1939 ya había dos ediciones internacionales de las tablas posteriores a la alemana de 1934: La 4a edición in ternacional de 1936 en len gua in glesa y la 5 a in ter na cio nal de 1939 en len gua francesa. Dictaminó que la clasificación de los fon dos de las bi blio te cas pú bli cas se adap taraal "Sis te ma De ci mal Mel vil Dewey mo dificado por el Instituto Internacional de Biblio gra fía de Bru se las y con las co rrec cio nes introducidas por su representación en Berlín”. En de fi ni tiva, en la ci ta da Or den, pri mó la re la ción en tre Es paña y Ale ma nia más que una po lí ti ca bi blio te ca ria que aca ta ra las recomendaciones internacionales en esta temá ti ca. Con todo ello, la Or den de 1939 ayudó a unificar las tareas técnicas, y su cum pli mien to, aun que ya se ve nía prac ti cando, se generalizó rápidamente.

En la ali dad se da una im plan ta ción to tal de la CDU en las bibliotecas españolas, aunque la si tua ción a par tir de 1989 ha cam bia do, por la disposición derogatoria de la CDU de 19 de mayo de 1989 , me dian te la cual no se im po ne la obli ga ción del em pleo del Sis te ma De ci mal para clasificar los fondos y los catálogos de las biblio te cas públicas (aun que pu die ra existir cier ta obli ga to rie dad se gún se de ri va de la aplicación en este caso del Derecho transitorio). Por tan to, exis te li ber tad por par te de los bibliotecarios españoles de implantar un siste macualesquieraclasificatorioen susbibliote cas, pues to que exis te un va cío le gal a par tir de esta disposición derogatoria y de la aprobación del Reglamento de bibliotecas públicas del Es tado de 1989. En este úl ti mo se especifica que las bibliotecas estarán sujetas a

36 Redactó y ofreció a la aprobación ministerial numerosas disposiciones como la Orden de 19 de septiembre de 1938, mediante la cual se anuló la prohibición de permitir a los lectores la consulta directa a los ficheros; la Orden de 20 de junio de 1938, que reguló el enseñar al niño el uso de las bibliotecas; la Orden de 24 de abril de 1939 , a través de la cual se creó el entonces Museo Arqueológico y después denominado Museo de América; La Orden de 13 de octubre de 1938, por la que se reformó el Depósito Legal; La Orden de 11 de enero de 1939, relativa a la organización de la Junta de adquisición de libros. El reconocimiento de su tarea bibliotecaria le llevó a que, finalizada la guerra, entrara en las ciudades inmediatamente después de las tropas representando al Ministerio de Educación Nacional. Su labor legislativa la desarrolló fundamentalmente en Vitoria y estuvo mediada por su formación teórica basada más en libros extranjeros que en un conocimiento global de la realidad bibliotecaria española.

37 La Clasificación Decimal Universal, traducción abreviada precedida por una introducción sobre el concepto y misión de biblioteca, con una reseña sobre los principios clasificaciones y la exposición del sistema.- San Sebastián : Editorial Internacional, 1942.

38 La traducción que hizo Lasso de la Vega disfrutó de ser la obra más utilizada hasta que en 1953 se publicó la edición oficial por el Instituto de Racionalización del Trabajo. Esta edición y la obra de Méndez Albarrán fueron durante un período prolongado de tiempo las obras más utilizadas en España, no sólo para el estudio de la CDU, sino también como manual de uso de la misma en las bibliotecas españolas 
las nor mas que dic te el nue vo con se jo Co or dinador de Bibliotecas A esta situación cabe añadir la concesión por parte de la Administra ción cen tral de com pe ten cias a las dis tin tas Au to no mías, en lo que res pec ta a las bi blio tecas es ta ta les allí ubi ca das y a las nor mas técni cas rec to ras en las mis mas.
Aunque actualmente se da una implantación total de la CDU, a partir de 1989 ésta puede su pri mir se o con vi vir con otros nue vos sis temas. Ello vie ne provoca do por la ine xis ten cia en el pla no le gal de un sis te ma cla si fica to rio. Esto presenta un aspectopositivo al evitarla rigidez y posibilitar la adopción, sin necesi- dad de acudir a medidas legales, de nuevos sis te mas fru to de la in ves ti ga ción que se está produ cien do. Sin em bar go, el va cío le gal presenta el inconveniente de favorecer una descolonización de las bibliotecas.

EGUIA, Ju lián de.MiBiblioteca.Clasificación. Bil bao : La Viz caína, 1920.

\section{BIBLIOGRAFIA}

ANDRES, Te re sa de.Indicacionesparala organizacióndelasbiblio te cas defrentes, cuarteles y hospitales. Valencia: Cultura Popular, 1937.

BULLETIN de l'Institut Internationalde Bibliographie.-1895-1916

CAS TILLOY QUIJADA, Ma nuel.“Una gran ad qui si ción para bi blio gra fía mo der na. La Cla si ficación De ci mal Dewey”. En:BOLETIN deArchivos, bibliotecasy Mu seos.- 1986, año I, n. 56; p. 68-72.

"Sis te mas de Cla si fi ca ción. Al Sr. D. Agus tín Bu llón de la To rre exdi pu ta do a Cor tes y pro mo tor de las le yes de 30 de ju nio y 29 de ju lio de 1984". En BOLETIN deArchivos, Bibliotecasy Museos. -1986, año I. n.7

"La Clasificación Decimaly lanomen claturabibliográfica"/InstitutoInternacional de Bibliografía; traducido por Manuel Castillo. En: BOLETIN de Archivos, Bibliotecas y Museos. -1896, año I, n. 8; p. 129-136

"Ta blas ge ne ra les de la Cla si fic ca ción De ci mal Univer sal". En:REVISTA de Archivos, Bibliotecas y Museos. -1897.

LaCla sificación bibliográfica Decimal, exposicióndelsistemaytraduccióndirec ta de las ta blas delmis mo. Salamanca [s.n.].-1897.

CASTRILLO, José Ma. "Ca tálo go por ma te rias". En:REVISTA de Archi vos, Biblio te cas y Mu seos, 1923, t. XLIV; p. 553-554 (comunicación presentada a la Asamblea de 1923).

CODORNIU, Ri car do. Cla sifica ción biblio gráfica de cimaly ex trac to de las ta blas em plea das en el Reperto rio Biblio gráfico Univer salparael uso delpersonal Facultativode Montes. -Madrid: Im pren ta Ale ma na, 1905.

"IMPORTANCIA de la adopción de un idioma internacional auxiliar para el progreso de científico".En: CONGRESO de la Aso cia ción es pa ño la para el pro gre so de la cien cia.- Zaragoza, 1908.

CULTURA POPULAR (Valencia). Sección de Bibliotecas. Realizaciones de la Es paña Leal: La Sec ción de bi blio te cas de Cul tu ra Po pular : un año de tra ba jo, ju lio 1936-ju lio 1937. Valen cia: Cul tu ra Po pu lar, 1938.

CHOUSA, Ca mi lo. Biblioteconomía, sistemasdeclasificación. Ma drid: Es cue la Su pe rior de Magisterios, 1927.

DIEZlozano, Baldomero. Cur sode Bibliología. Mur cia [s.n.] 1928.

DOMINGUEZBORDONA, L. Je sús “La im plan ta ción de la Cla si fi ca ción De ci mal Uni ver sal”. En REVISTA de Archivos, Biblio te cas y Mu seos.- 1923, t. XLIV ; (Co mu ni ca ción no. 7 pre sentada a la Asamblea de 1923).

DURAN, Félix. "Los catálogos de materias". En: REVISTA de Archivos, Bibliotecas y Museos. 1923, T. XLIV; p. 557-559. 
ESPAÑA. Mi nis te rio de Ins truc ción Pública. Con se jo Cen tral de Ar chi vos, Bi blio te cas y Te soro Artís tico. Sección de Biblio te cas.Instruccionespara el Servicio de pequeñas Bibliote cas / [Por Ma ría Mo li ner] En: La LECTURA Pública en Es pa ña, Op. cit.; p. 19.

FARNES, Se bas tián. La cla sifica ción de la Biblio te ca del Fo men to del TrabajoNacional. Bar ce lo na: [s.n.], 1914.

FAUS SEVILLA, Pi lar. La lec tu ra pública en Es paña y el Plan de biblio te cas de María Moliner.- Madrid: ANABAD, 1990.

FER NANDEZ-VICTORIOY PEREIRA, Ni co lás. La clasificación por materias enlasbibliotecas españolas. Madrid: Ins ti tu to Ni colás An tonio, CSIC, 1944.

"Consideraciones sobre la colocación de los libros en las bibliotecas". En: BOLETIN delaDirección GeneraldeArchivosyBibliotecas. 1957, 42.

FILL, Karl. Introducción al empleo y métodos de la Clasificación Decimal Universal. Ber lín : [s.n.] 1965.

FONSECA, Isa bel. "La CDU en Es pa ña”. En:BOLETIN de la ANABAD. 1978, año XVII, n. 2; p. 3-24.

GARCIARIVES, Luis. "El bi blio te ca rio y sus fun cio nes téc ni cas". En:RE VISTA de Archivos, Bibliote cas yMu seos.- 1923, t. XLIV; p. 619-622 (comunicación presentada a la Asamblea de 1923).

GARCIA SORIANO, Justo. Bibliotecas: obra ajustada al cuestionario de temas de 23 de no viem bre de 1924 para el ejer ci cio teó ri co de las opo siciones al Cuerpo Facultativo de Archiveros, Bibliotecarios y Arqueólogos. Ma drid : Reus, 1930.

GIMENEZ, Leo pol do. No ticia so breel servicio de In for ma ción bi blio gráfica es table cidoen la Bibliote cade In ge nie rosdelEjército. Ma drid : [s.n.], 1906.

GIMENO PERELLO, Javier. "Clasificación. Clasificaciones jerárquicas. La CDU." En: OPE RA CIO NES de la ca de na do cumen tal: Uni dad di dáctica.- Madrid : Instituto Ofi cial de Ra dio y Te le vi sión, D.L. 1988.

GOMEZVILlafRANCA, Ro mán. Catálogo de la Re vis ta y el Bo le tín de Archi vos, Biblio te cas y Mu seos en sus tresépo cas (ene ro de 1871, diciembrede 1910)formadoaplicandola Cla sificación Decimal. Ma drid : tip. de la Rev., 1911.

HINOJOSA, Ri car do de. "Jui cio so bre las ins truc cio nes". En REVISTA de Archivos, BibliotecasyMuseos. 1924, julio, T-XI, 3a. época; p. 26-50.

Historia de España/dirigida por Manuel Tuñón de Lara. Madrid : Labor, 1980-1981. T. VII y VIII.

Ins trucciones para la for ma ción de los ca tálo gos y con servación de los libros en la biblioteca de la Academia de Ingenieros del Ejército.- Madrid [s.n.], 1906.

LASSO DE LA VEGA, Ja vier. "Políticabiblio te caria". En:BOLETIN de Bi bliotecas ybiblio grafía. 1934, I; p. 10.

Cómo utilizarunabiblioteca. Ma drid [s.n.], 1935.

Re glas paralaforma ción de los ca tálo gos dic cio narios de las bibliotecas. Vitoria [s.n.], 1939. 
La Clasificación Decimal Universal, traducción abreviada precedida por una in tro duc ción so bre el con cep to y mi sión de bi blio te ca, con una reseña sobre las principalesclasificaciones y la exposición del sistema. San Se bas tián : Edi to rial In te na cio nal, 1942.

"Nue vas con si de ra cio nes so bre la co lo ca ción de los li bros en los de pó si tos". En: BOLETIN de la Dirección Ge ne ralde Ar chi vos y Biblio te cas. 1947 , 44 .

"LA CDU como me dio para ha cer sis te má ti cas las lis tas de epí gra fes de los catá lo gos de asun tos". En: REVIEW ofDocumentation. La Haya, 1960; p. $169-170$

"Cla si fi ca ción de la pe da go gía y el mer ca do de las ideas". En: RACIONALIZACION. 1971, marzo.

Los relacionadores: un avance para la clasificación en profundidad de la $C D U$. Madrid :Asociación Nacional de Bibliotecarios y Arqueólogos (Homenaje a Fe de rico Navarro), 1973. - p. 227-235.

Cómo se hace una te sis doc to ral: (Ma nual de do cu men ta ción). Ma drid : Fundación Univesitaria española, 1977.

La ver da de ra his to ria de la Cla sifica ción De ci malde Dewey. Ma drid [s.n.], 1979.

LOPEZ YEPES, José; SAGREDO, F. Es tudios de do cu men ta ción ge ne ral e informativa. - Ma drid : Se mi na rio Mi lla res Car lo, 1981.

MA TEU Y LLO PIS, Fe li pe. "Or de na ción bibliográfi ca de la cien cia es pa ñola". En: REVISTA de Archivos, bibliotecas y Museos. 1956.T. LXII.

MA TEU IBARS, Jo se fi na. Bibliografía paleográfica. Barce lo na: Fa cul tad de Fi lo so fía y Le tras, 1974.

MENDEZALBARRAN, Luis. LaClasificación Decimal, exposición del sistema y sus tablas compendiadas.- Badajoz: [s.n.], 1931.

MOURI LLO, M.F. "El ca tá lo go por con cep tos". En:REVISTA de Ar chi vos, Bibliotecas ymu seos.- 1907, I; p. 252-255.

OR TE GA Y GAS SET, José. Lamisión delbiblio te ca rio. Ma drid : Edi cio nes de la Re vis ta de Oc ci den te, 1967.

PAZ Y ME LIA, An to nio "La cues tión de las Bi blio te cas Na cio na les y su di fusión de la cultura". En: REVISTA de Archivos, Bibliotecas y Museos, 1910 ; p. 109 .

REVISTAdeArchivos, Biblio tecas y Museos: de dicadal Cuerpo Facultativo del ramo.- Madrid.

1a.época 1871-1878 (Anuario 1881-1882)

2a.época 1883 -Boletín 1896 (En 1895 se suspende la publicación)

3a. época 1897-1931

4a. época 1947-1953

5a. época $1953-1980$

RUBIOIBALA GUER, Jor di. Clas sifica ció de ci mal adap ta cióperales Biblioteques Populars de la Mancomunitat de Catalunya.- Barcelona:Casa Caritat, 1921.

Cómoseorganizaycatalogaunabiblioteca. Barce lo na: Cá ma ra Ofi cial al Li bro 1932 . 
Catalogación y ordenación de biblitecas. Instrucciones elementales. Barcelona: Tabor, [1928?]

Los libros y las biblio te cas, una cartilla para suordenación. Barce lo na: Gremio de edi to res y li bre rías, 1952.

RUBIO I LOIS Jordi. Homenatge a Jordi Rubió i Lois, inauguració del curs academic 1988-1989.

RUBIOY CAMBRONERO, Ig na cio. "El li bre ac ce so a los es tan tes en las bi bliotecas". En: REVISTA de Archivos, Bibliotecas y Museos. 1923, T.XLIV; p. 553.

RUIZ CABRIADA, Agustín, Bio-Bibliografía del Cuerpo Facultativo de Archiveros, Biblio te carios y Arqueólo gos 1858; 1958. Ma drid: Jun ta técnica de Archivos, Bibliotecas y Mu seos, 1958.

SAN SIMON, José de. "Los ca tá lo gos de las bi blio te cas". En: REVISTA de Archivos, bibliotecasyMuseos, 1923, XXVIII; p. 554.

VICENS, Juan. L'Espag ne vi van te: un peup le à la con quê te de la Cul ture. París: Editions Sociales Intenationales, 1938.

Manual del Catálogo-Diccionario. México : Atlanta, 1942.

Cómoorganizarbibliotecas. México : Atlan ta, 1946.

ZAMBRANO, Ma ría. Los in te lec tua les en el dra ma de Es pa ña: en sa yos y notas (1936-1939). Madrid : [s.n.], 1977. 